\title{
A mortalidade por doenças infecciosas e parasitárias na população idosa brasileira
}

\author{
Neir Antunes Paes ${ }^{1}$
}

Como citar Paes NA. A mortalidade por doenças infecciosas e parasitárias na população idosa brasileira. Rev Panam Salud Publica. 2004;15(4):233-41.

RESUMO Objetivo. Traçar o perfil da mortalidade por doenças infecciosas e parasitárias na população de mais de 65 anos no Brasil e nas unidades da federação e investigar sua associação com determinadas variáveis socioeconômicas no período de 1980 a 1995.

Métodos. Foram utilizados os dados do Sistema de Informações sobre Mortalidade. Foram calculadas taxas de mortalidade padronizadas para tuberculose, tripanossomíase e septicemia, assim como a participação relativa dessas causas na mortalidade geral. Analisou-se a relação entre as taxas de mortalidade por doenças infecciosas e parasitárias e sexo e as seguintes variáveis socioeconômicas: produto interno bruto per capita; número de leitos hospitalares por habitante; relação entre a população urbana e a total; número de benefícios em manutenção pela previdência social por habitante; coeficiente de letalidade; e esperança de vida ao nascer. Para esta análise foi utilizado o modelo normal de regressão linear múltipla.

Resultados. Não houve alteração significativa no percentual de óbitos por doenças infecciosas e parasitárias na população idosa brasileira no perído para ambos os sexos. Por outro lado, verificou-se uma importante transição no período, caracterizada pela diminuição da participação da tuberculose pulmonar e da tripanossomíase e pela tendência de aumento na participação da septicemia para ambos os sexos. A análise ecológica mostrou que os indicadores socioeconômicos analisados tiveram baixo poder explicativo para os diferentes padrões de mortalidade nas diferentes regiões do país.

Conclusões. Os presentes resultados sugerem uma estagnação das políticas de prevenção $e$ combate às doenças infecciosas e parasitárias como um todo. Essa observação, juntamente com a progressiva pressão populacional dos idosos, indica que os niveis de mortalidade por doenças infecciosas e parasitárias não deverão diminuir nessa faixa etária, pelo menos em um futuro próximo.

Palavras-chave Análise estatística, doença de Chagas, idoso, saúde do idoso, septicemia, tuberculose pulmonar.

Nas últimas décadas, o declínio da fecundidade, juntamente com um

\footnotetext{
Universidade Federal da Paraíba, Departamento de Estatística. Correspondência e pedidos de separatas devem ser enviados ao seguinte endereço: Departamento de Estatística, Centro das Ciências Exatas e da Natureza, Universidade Federal da Paraíba, Campus I, CEP 58051-910, João Pessoa, PB, Brasil. Fax: +55-83-2167117; e-mail: antunes@ de.ufpb.br
}

maior controle de várias doenças potencialmente fatais, vem conduzindo a um aumento da expectativa de vida e conseqüente envelhecimento da população brasileira. No país, a participação dos idosos (65 anos e mais) na população cresceu de 6,1\% em 1980 para 7,4\% em 1991 e 8,1\% em 1996. A previsão para 2010 é de que este grupo etário seja equivalente a 9,2\% da população brasileira (1). Para 2025, um estudo (2) sugere que o grupo de mais de 60 anos deverá ser 15 vezes maior do que em 1950, contra um aumento de cinco vezes para a população como um todo.

Nesse processo de transição demográfica, o perfil da mortalidade da po- 
pulação brasileira vem sendo substancialmente modificado, em virtude de uma política pública mais eficiente de prevenção e combate a um grupo de doenças evitáveis, especialmente doenças infecciosas e parasitárias (DIPs). Tal política se aplica prioritariamente à população infantil, na qual as DIPs exercem um importante papel (3). Em relação à população de mais de 60 anos, contudo, é incipiente o enfoque nas DIPs, embora seja possível especular que nos países em desenvolvimento sua presença neste grupo etário ainda ocupe destaque.

Chackiel (4) chamou a atenção para a existência de poucos estudos de mortalidade por causas na América Latina, e argumentou que tal fato estaria provavelmente associado à pouca qualidade dos dados oficiais. No Brasil, mais raros ainda são os estudos associando a mortalidade por causas com fatores de natureza social e econômica (5-9). Mesmo assim, acredita-se que os dados disponíveis nos sistemas nacionais de informação guardem um potencial explicativo suficiente para se traçar um perfil satisfatório das tendências, padrões e diferenciais de mortalidade no país, além de permitir especular sobre as relações estatísticas que essa mortalidade possa ter com certos fatores sociais e econômicos.

Desse modo, o objetivo do presente estudo foi traçar o perfil das DIPs na população de mais de 65 anos no Brasil e em todas as unidades da federação no período de 1980 a 1995, bem como investigar a associação entre essa causa de morte e um conjunto de indicadores de natureza socioeconômica.

\section{MATERIAIS E MÉTODOS}

Utilizou-se como fonte primária de dados o CD-ROM do Sistema de Informações sobre Mortalidade (SIM), desenvolvido e implantado no Brasil pelo Ministério da Saúde em 1975 (10). Para obter taxas de mortalidade mais precisas, decidiu-se limitar este estudo pelos anos censitários de 1980 e 1991 e pela contagem populacional de 1996 (11-13), cujas taxas referem-se aos triênios de 1979 a 1981 e de 1990 a 1992 e ao ano de 1995, sendo os dados desagregados por sexo e grupos etários qüinqüenais de 65 anos em diante.

Para permitir a comparação das taxas de mortalidade entre os sexos, as unidades da federação e os anos, tomou-se como padrão a estrutura etária da população brasileira total em 1991. Para determinar a contribuição dos percentuais de óbitos por DIPs agregou-se o triênio de 1984 a 1986. Trabalhou-se, então, com a média dos triênios, com o intuito de minimizar o efeito de eventuais flutuações aleatórias dos dados. O ano de 1995 foi mantido isolado em virtude de sérios problemas de consistência dos dados de óbitos para 1996 e pela proximidade do ano de 1994 com o ano de 1992, o qual foi utilizado no triênio de 1990 a 1992. Para possibilitar a análise dos óbitos por DIPs foi adotada a nona revisão da classificação internacional de doenças (CID-9) (a CID-10 não foi utilizada pois sua classificação é válida somente a partir de 1996). A CID-BR, uma adaptação da CID-9 para a realidade brasileira, também foi utilizada (10). Por sua importância relativa dentro do grupo das DIPs, foram analisadas as seguintes doenças: a tuberculose pulmonar (código CID-9: 020), a septicemia (código CID-9: 038) e a tripanossomíase (código CID-9: 054).

É inevitável o questionamento sobre a confiabilidade dos registros e declarações de óbitos no Brasil. Segundo Paes e Albuquerque (14), em 1980 o percentual de óbitos com "causa básica desconhecida" (resultante da combinação do percentual de sub-registro com o de causas mal definidas) foi da ordem de $38 \%$ para os homens e de $44 \%$ para as mulheres. Em 1990, esse percentual ficou em $27 \%$ para homens e $30 \%$ para mulheres. Apesar da melhora, ainda persiste um certo desconforto estatístico, acentuado pela presença da septicemia como causa básica de morte, já que esta causa é, na verdade, decorrente de uma doença complicada por infecção generalizada, e não uma doença em si mesma - o que possivelmente compromete a fidedignidade do real perfil da mortalidade, independentemente da faixa etária (15).
Tendo em vista esses aspectos, lançou-se mão de um sistema que classifica as unidades da federação de acordo com uma estimativa do percentual de óbitos com causa básica desconhecida. Para a população masculina, cinco grupos foram identificados: unidades da federação com percentual de óbitos com causa básica desconhecida de 0 a 15\%, 16 a 30\%, 31 a 50\%, 51 a $75 \%$ e mais de $75 \%$. Para a população feminina, foram considerados apenas os quatro primeiros grupos. A classificação mostrou uma forte associação entre o nível de desenvolvimento econômico das unidades da federação e a qualidade dos registros de óbitos. Nesse sentido, os estados com melhor qualidade foram os das regiões Sul e Sudeste, seguidos pelos da Região Centro-Oeste.

Com relação à associação entre as taxas de mortalidade por causas de morte e fatores de natureza socioeconômica, foram considerados, com base em diversas investigações (1619), os seguintes indicadores ou variáveis: produto interno bruto per capita, que é a razão entre o somatório da renda pessoal de todos os indivíduos e o número total de indivíduos na unidade familiar (20); número de leitos hospitalares por habitante $(21,22)$; relação entre a população urbana e a total (11-13); número de benefícios em manutenção pela previdência social por habitante (23); coeficiente de letalidade, que representa o número de óbitos informados para cada 100 internações $(21,22)$; e esperança de vida ao nascer, que é dada pelo número médio de anos que as pessoas viveriam a partir do nascimento (20).

Para estudar a relação desses indicadores com as taxas de mortalidade, por DIP e por sexo, para todas as unidades da federação, utilizou-se o modelo normal de regressão linear múltipla. Posteriormente, foi usado o procedimento em etapas (stepwise), que consiste na escolha de uma melhor equação de regressão, o que, no presente estudo, foi feito pelo processo retrógrado (backward) que, por sua vez, consiste na retirada de variáveis até se chegar ao mais adequado modelo final de regressão $(24,25)$. Nos ajustes reti- 
raram-se os estados do Acre, Rondônia, Roraima e Amapá (com qualidade deficiente dos dados) e o Distrito Federal, por apresentarem problemas de ajustamento. Assim, os resultados dos ajustes dos modelos de regressão múltipla não contemplam esses estados.

\section{RESULTADOS}

Não houve alteração significativa no percentual de óbitos por DIPs na população idosa brasileira no período de 1980 a 1995, para ambos os sexos (tabela 1). As doenças do aparelho circulatório e os neoplasmas responderam pelos mais altos percentuais de óbitos, excluindo-se a causa dos sintomas, sinais e afecções mal definidas. Entre mulheres houve um discreto aumento sistemático tanto no percentual de óbitos por DIPs, que cresceu de 2,1\% em
1980 para 2,5\% em 1995, quanto na taxa de mortalidade, que passou de 96,6 em 100000 mulheres em 1980 para 110,1 em 1995. Apesar disso, a presença das mortes por DIP foi mais importante para os homens durante todo o período. Como as idosas vivem mais e são mais numerosas, estão mais expostas à competição dos riscos de morte, perdendo importância as mortes por DIPs.

Quando se analisam os percentuais de óbitos por DIPs em termos dos grupos de idade (tabela 2), observa-se uma tendência de decréscimo para ambos os sexos. Apesar desse padrão, os níveis perderam sua força de declínio no tempo a partir dos 80 anos para os homens e dos 70 anos para as mulheres. Mais chamativos ainda são os indícios de que o ritmo de declínio relativo no tempo parece ter-se desacelerado, ou seja, a redução por idade foi muito mais acentuada em 1980 do que em
1995. Esses comportamentos sugerem uma deterioração do controle das doenças infecciosas no país para os idosos ao longo do tempo, e uma maior dificuldade em reduzir os níveis da mortalidade nas idades mais avançadas.

Se o panorama do país sugere uma estagnação dos níveis de mortalidade pelas doenças infecciosas e parasitárias no período de 1980 a 1995, em nível regional as tendências variaram. Para a análise do comportamento das DIPs como um todo, e do comportamento da tuberculose pulmonar, tripanossomíase e septicemia, foram excluídos alguns estados da Região Norte (Rondônia, Roraima, Amapá e Acre), devido a inconsistências nos dados originais. Como o total da população desses estados representa menos de $5 \%$ da população total do Brasil, a desconsideração de tais resultados não impede uma visão panorâmica do país.

TABELA 1. Mortalidade proporcional e taxas de mortalidade padronizadas por sexo das principais causas de óbito, Brasil

\begin{tabular}{|c|c|c|c|c|c|c|c|c|c|}
\hline \multirow[b]{2}{*}{ Causas de óbito } & \multicolumn{4}{|c|}{ Óbitos (\%) } & \multicolumn{3}{|c|}{ Taxa de mortalidade ${ }^{a}$} & \multicolumn{2}{|c|}{$\begin{array}{c}\text { Variação da taxa de } \\
\text { mortalidade (\%) }\end{array}$} \\
\hline & 1980 & 1985 & 1991 & 1995 & 1980 & 1991 & 1995 & $1980 / 1991$ & $1991 / 1995$ \\
\hline \multicolumn{10}{|l|}{ Homens } \\
\hline Doenças infecciosas e parasitárias & 2,60 & 2,54 & 2,61 & 2,63 & 150,54 & 157,47 & 150,96 & $-0,05$ & 0,04 \\
\hline Tuberculose pulmonar & 0,66 & 0,46 & 0,39 & 0,33 & 36,90 & 23,51 & 24,89 & 0,57 & $-0,05$ \\
\hline Septicemia & 0,26 & 0,47 & 0,68 & 0,56 & 15,94 & 41,68 & 44,13 & $-0,62$ & $-0,05$ \\
\hline Tripanossomíase & 0,70 & 0,63 & 0,61 & 0,42 & 39,12 & 36,21 & 31,77 & 0,08 & 0,14 \\
\hline Neoplasmas & 11,67 & 11,40 & 12,76 & 13,54 & 677,03 & 764,40 & 769,76 & $-0,13$ & $-0,01$ \\
\hline \multicolumn{10}{|l|}{ Doenças endócrinas, nutricionais, metabólicas } \\
\hline e transtornos imunitários & 2,43 & 2,65 & 3,30 & 3,63 & 145,94 & 199,34 & 208,41 & $-0,37$ & $-0,05$ \\
\hline Doenças do aparelho circulatório & 43,34 & 39,86 & 38,49 & 36,98 & 2669,23 & 2334,11 & 2127,10 & 0,13 & 0,09 \\
\hline Doenças do aparelho respiratório & 7,75 & 9,38 & 11,21 & 12,95 & 489,29 & 686,32 & 751,73 & $-0,40$ & $-0,10$ \\
\hline Doenças do aparelho digestivo & 3,30 & 3,34 & 3,66 & 3,77 & 192,87 & 220,25 & 215,43 & $-0,14$ & 0,02 \\
\hline Sintomas, sinais e afecções mal definidas & 23,32 & 25,41 & 22,13 & 20,20 & 1478,81 & 1358,45 & 1181,62 & 0,08 & 0,13 \\
\hline Causas externas & 2,91 & 2,85 & 2,86 & 3,04 & 170,50 & 171,69 & 173,42 & $-0,01$ & $-0,01$ \\
\hline Demais causas & 2,72 & 2,56 & 2,96 & 3,25 & 166,44 & 179,54 & 188,04 & $-0,08$ & $-0,05$ \\
\hline \multicolumn{10}{|l|}{ Mulheres } \\
\hline Doenças infecciosas e parasitárias & 2,09 & 2,32 & 2,42 & 2,54 & 96,59 & 102,59 & 110,10 & $-0,06$ & $-0,07$ \\
\hline Tuberculose pulmonar & 0,31 & 0,20 & 0,17 & 0,14 & 14,19 & 7,24 & 8,19 & 0,97 & $-0,12$ \\
\hline Septicemia & 0,27 & 0,54 & 0,78 & 0,67 & 12,79 & 32,69 & 38,18 & $-0,61$ & $-0,14$ \\
\hline Tripanossomíase & 0,57 & 0,54 & 0,52 & 0,38 & 26,12 & 22,31 & 22,03 & 0,17 & 0,01 \\
\hline Neoplasmas & 9,66 & 9,42 & 10,61 & 11,15 & 445,11 & 453,80 & 490,15 & $-0,02$ & $-0,08$ \\
\hline \multicolumn{10}{|l|}{ Doenças endócrinas, nutricionais, metabólicas } \\
\hline e transtornos imunitários & 4,02 & 4,53 & 5,44 & 5,99 & 187,70 & 231,11 & 260,59 & $-0,23$ & $-0,13$ \\
\hline Doenças do aparelho circulatório & 47,94 & 43,88 & 42,35 & 40,51 & 2281,77 & 1783,21 & 1742,54 & 0,22 & 0,02 \\
\hline Doenças do aparelho respiratório & 6,53 & 8,11 & 9,54 & 11,15 & 313,03 & 399,16 & 475,41 & $-0,28$ & -019 \\
\hline Doenças do aparelho digestivo & 2,84 & 2,96 & 3,27 & 3,46 & 132,49 & 138,41 & 149,59 & $-0,04$ & $-0,08$ \\
\hline Sintomas, sinais e afecções mal definidas & 22,80 & 24,81 & 21,90 & 20,46 & 1089,47 & 914,60 & 870,21 & 0,16 & 0,05 \\
\hline Causas externas & 1,77 & 1,71 & 1,78 & 1,75 & 82,97 & 75,13 & 75,78 & 0,09 & $-0,01$ \\
\hline Demais causas & 2,36 & 2,26 & 2,70 & 2,99 & 111,04 & 113,90 & 128,77 & $-0,03$ & $-0,13$ \\
\hline
\end{tabular}

Fonte: CD-ROM do Sistema de Informações sobre Mortalidade (SIM) do Ministério de Saúde do Brasil, 1979 a 1997.

a Óbitos por 100000 habitantes. 
TABELA 2. Contribuição percentual das doenças infecciosas e parasitárias, tuberculose, tripanossomíase e septicemia para a mortalidade por grupos etários e sexo, Brasil, 1980 a 1995

\begin{tabular}{|c|c|c|c|c|c|c|c|c|c|c|}
\hline & \multicolumn{5}{|c|}{ Grupos etários masculinos } & \multicolumn{5}{|c|}{ Grupos etários femininos } \\
\hline & 65 a 69 & 70 a 74 & 75 a 79 & 80 a 84 & 85 ou mais & 65 a 69 & 70 a 74 & 75 a 79 & 80 a 84 & 85 ou mais \\
\hline \multicolumn{11}{|c|}{ Doenças infecciosas e parasitárias } \\
\hline 1985 & 3,23 & 2,78 & 2,39 & 2,03 & 1,79 & 3,10 & 2,56 & 2,36 & 2,04 & 1,64 \\
\hline 1991 & 3,15 & 2,78 & 2,52 & 2,33 & 2,03 & 2,97 & 2,64 & 2,44 & 2,19 & 2,04 \\
\hline 1995 & 2,97 & 2,80 & 2,42 & 2,48 & 2,34 & 3,04 & 2,68 & 2,65 & 2,38 & 2,13 \\
\hline 1985 & 0,75 & 0,59 & 0,47 & 0,28 & 0,19 & 0,40 & 0,30 & 0,21 & 0,13 & 0,09 \\
\hline 1991 & 0,64 & 0,50 & 0,43 & 0,26 & 0,19 & 0,34 & 0,21 & 0,20 & 0,14 & 0,10 \\
\hline 1995 & 0,46 & 0,43 & 0,33 & 0,27 & 0,17 & 0,25 & 0,20 & 0,16 & 0,13 & 0,08 \\
\hline \multicolumn{11}{|c|}{ Tripanossomíase } \\
\hline 1980 & 1,08 & 0,80 & 0,62 & 0,38 & 0,22 & 1,09 & 0,78 & 0,53 & 0,28 & 0,13 \\
\hline 1985 & 1,01 & 0,74 & 0,56 & 0,38 & 0,22 & 1,05 & 0,73 & 0,52 & 0,35 & 0,15 \\
\hline 1991 & 0,97 & 0,72 & 0,53 & 0,40 & 0,27 & 0,91 & 0,74 & 0,55 & 0,33 & 0,21 \\
\hline 1991 & 0,63 & 0,70 & 0,69 & 0,71 & 0,71 & 0,74 & 0,77 & 0,76 & 0,79 & 0,82 \\
\hline 1995 & 0,46 & 0,51 & 0,54 & 0,66 & 0,73 & 0,60 & 0,61 & 0,68 & 0,74 & 0,69 \\
\hline
\end{tabular}

Fonte: CD-ROM do Sistema de Informações sobre Mortalidade (SIM) do Ministério de Saúde do Brasil, 1979 a 1997.

A figura 1 revela que as taxas padronizadas de mortalidade por DIPs foram superiores para os homens em todas as unidades da federação, independentemente da classificação adotada. No entanto, os níveis da mortalidade foram mais exacerbados, em ambos os sexos, no Distrito Federal e em Goiás, tendo como principal responsável a tripanossomíase. Em nenhum dos demais estados (exceto Minas Gerais e Mato Grosso do Sul) os níveis de mortalidade para ambos os sexos ultrapassaram 200 óbitos por 100000 habitantes. Quanto à tendência, não se observou um comportamento evolutivo uniforme para todas as unidades da federação em ambos os sexos. Tanto nos estados mais desenvolvidos economicamente, em particular aqueles pertencentes aos dois primeiros grupos classificatórios, quanto nos menos desenvolvidos, houve uma tendência tanto de aumento como de diminuição das taxas no período. Essas tendências não parecem depender da qualidade das classificações adotadas, e sim de uma real tendência nos níveis da mortalidade. Nos estados com maior peso popula- cional no país, São Paulo e Rio de Janeiro, houve uma discreta elevação das taxas de mortalidade por DIPs.

\section{Tuberculose pulmonar}

No Brasil, pôde-se observar um acentuado declínio na participação da tuberculose pulmonar como causa de morte em ambos os sexos (tabela 1). Além disso, a tabela 2 mostra um declínio na proporção de mortes por tuberculose nos idosos de ambos os sexos à medida que se avança nas idades em todos os anos estudados. As maiores reduções no tempo ocorreram nas primeiras idades, o que sugere uma maior resistência à diminuição de sua incidência com o aumento da idade.

A figura 2 revela, para ambos os sexos, que os níveis da mortalidade foram decrescentes para a maioria dos estados e para o Distrito Federal, sendo que os níveis foram, para os homens, pelo menos o dobro do verificado para as mulheres na maioria dos estados. Essa redução sugere que a qualidade dos dados não interfe- riu nas reais tendências observadas, embora os níveis sejam obviamente subestimados. Apenas três estados tiveram aumento das taxas de mortalidade por tuberculose (Mato Grosso, Pernambuco e Mato Grosso do Sul), porém com taxa inferior a 20 óbitos por 100000 habitantes. As maiores reduções nas taxas de mortalidade verificaram-se nos estados de Minas Gerais, Paraná, Espírito Santo, Pará, Bahia e no Distrito Federal para ambos os sexos; em Mato Grosso do Sul para os homens; e em Mato Grosso, Pernambuco e Alagoas para as mulheres.

\section{Tripanossomíase}

A tabela 1 mostra que, para o país como um todo, a tripanossomíase, ou doença de Chagas, participava, em 1980, com o mais alto percentual de óbitos entre as principais causas, e que somente em 1991 essa doença perdeu em importância para a septicemia. As taxas de mortalidade também seguiram uma tendência de declínio. A tabela 2 mostra que a tripanossomíase teve um comportamento semelhante 
FIGURA 1. Taxas padronizadas de mortalidade das doenças infecciosas e parasitárias por sexo segundo classificação de causas de morte desconhecidas nas unidades da federação, Brasil, 1980 a $1995^{a}$
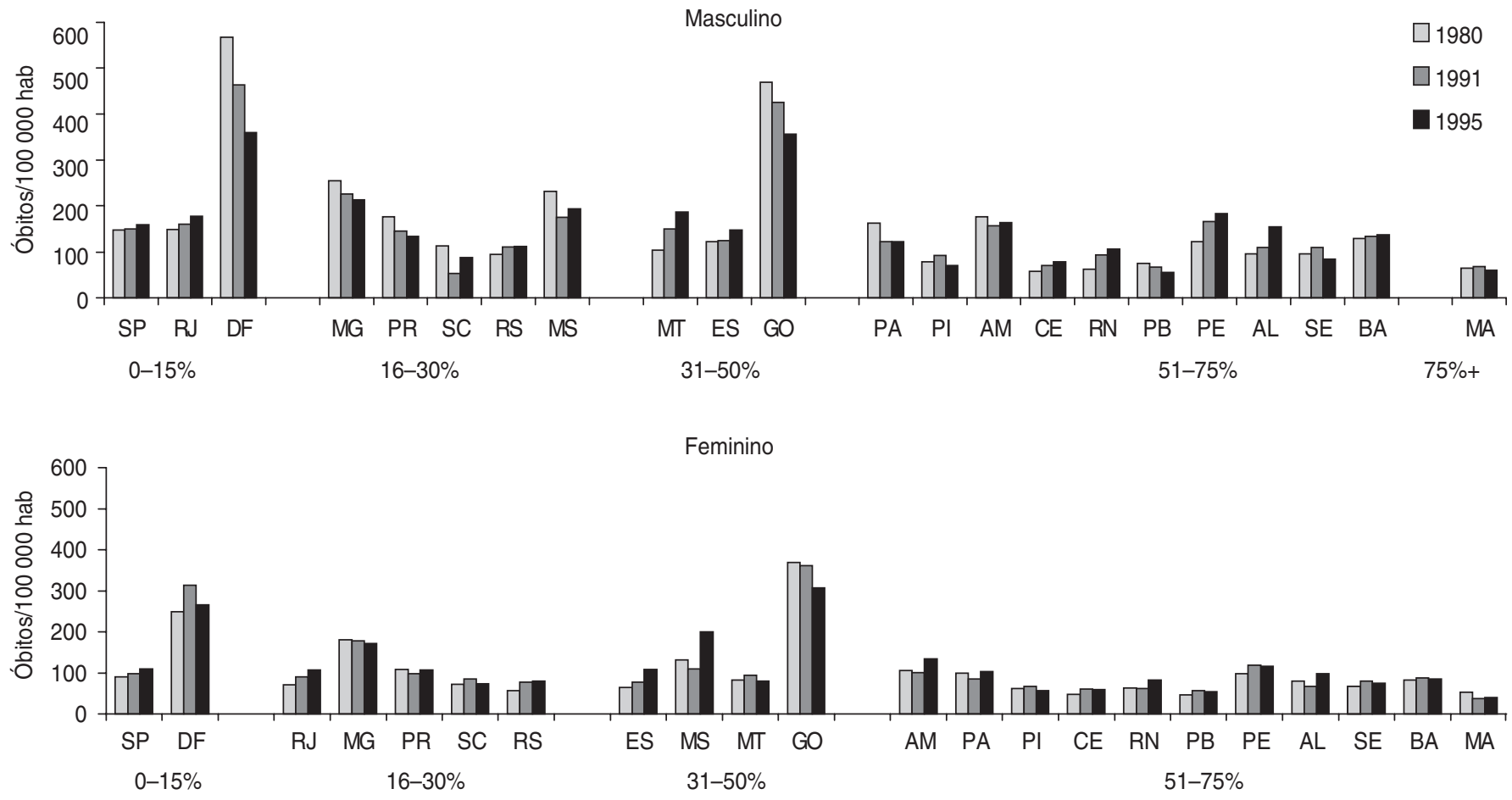

Grupos de estados conforme percentual de óbitos com causa básica desconhecida

${ }^{a} \mathrm{AL}=$ Alagoas; $\mathrm{AM}=$ Amazonas; $\mathrm{BA}=$ Bahia; $\mathrm{CE}=$ Ceará; $\mathrm{DF}=$ Distrito Federal; $\mathrm{ES}=$ Espírito Santo; $\mathrm{GO}=$ Goiás; $\mathrm{MA}=\mathrm{Maranhão} ; \mathrm{MG}=\mathrm{Minas}$ Gerais; $\mathrm{MS}=\mathrm{Mato}$ Grosso do Sul; $\mathrm{MT}=$ Mato Grosso; PA = Pará; PB = Paraíba; PE = Pernambuco; PI = Piauí; PR = Paraná; RJ = Rio de Janeiro; RN = Rio Grande do Norte; RS = Rio Grande do Sul; SC = Santa Catarina; SE = Sergipe; SP = São Paulo. Não foram considerados os estados de Rondônia, Roraima, Amapá e Acre (Região Norte). O Estado do Tocantins não consta pois foi criado em 1988.

ao apresentado pela tuberculose pulmonar, ou seja, de descensão proporcional quase linear por grupos etários. Embora com níveis mais elevados para os homens, o ritmo de descensão foi mais intenso para as mulheres.

A participação mais significativa da tripanossomíase nas taxas de mortalidade no período de 1980 a 1995 ocorreu no Estado de Goiás, com valores decrescentes em ambos os sexos, variando, no período, de 350 a 234 óbitos por 100000 habitantes no sexo feminino e de 299 para 189 óbitos no sexo masculino. Em seguida vieram o Distrito Federal e o Estado de Minas Gerais. Os demais estados não apresentaram participação expressiva nessa causa, em ambos os sexos, atingindo um máximo de 46 por 100000 habitantes em 1980 e de 39 por 100000 habitantes em 1995. Em geral, os níveis foram mais elevados para os homens.

\section{Septicemia}

A septicemia (tabela 1) foi a única das principais causas a apresentar aumento nos seus níveis de mortalidade em todo o período, de 0,26 para $0,56 \%$ para os homens e de 0,27 para 0,67\% para as mulheres. Quando se analisa seu comportamento por faixa etária observa-se um aumento de sua importância relativa à medida que aumenta a idade (tabela 2).

Das três principais causas de óbitos, a septicemia despontou como aquela que abrangeu o maior número de estados com elevação quase sistemática das taxas de mortalidade, abrangendo quase todo o país (figura 3). O aumento das taxas ocorreu independentemente da classificação adotada, indicando que, embora os níveis estejam subestimados, principalmente no Nordeste, a tendência foi clara. Com aumentos mais significativos para o sexo masculino, os níveis foram especialmente elevados em Mato Grosso do Sul, Minas Gerais, Rio de Janeiro e Goiás, estados considerados como mais desenvolvidos do que a maioria daqueles pertencentes às regiões Norte e Nordeste. Uma tendência de decréscimo no período de 1980 a 1991 ocorreu apenas para Santa Catarina. Porém, entre 1991 e 1995 houve uma pequena redução em alguns estados, o que poderia ser atribuído a um tolerável erro, inerente às magnitudes das taxas.

\section{Associação das doenças infecciosas e parasitárias com variáveis socioeconômicas}

Em um exercício especulativo, considerou-se o conjunto de variáveis e in- 
FIGURA 2. Taxas de mortalidade padronizadas da tuberculose pulmonar por sexo segundo classificação de causas de morte desconhecidas nas unidades da federação, Brasil, 1980 a $1995^{a}$

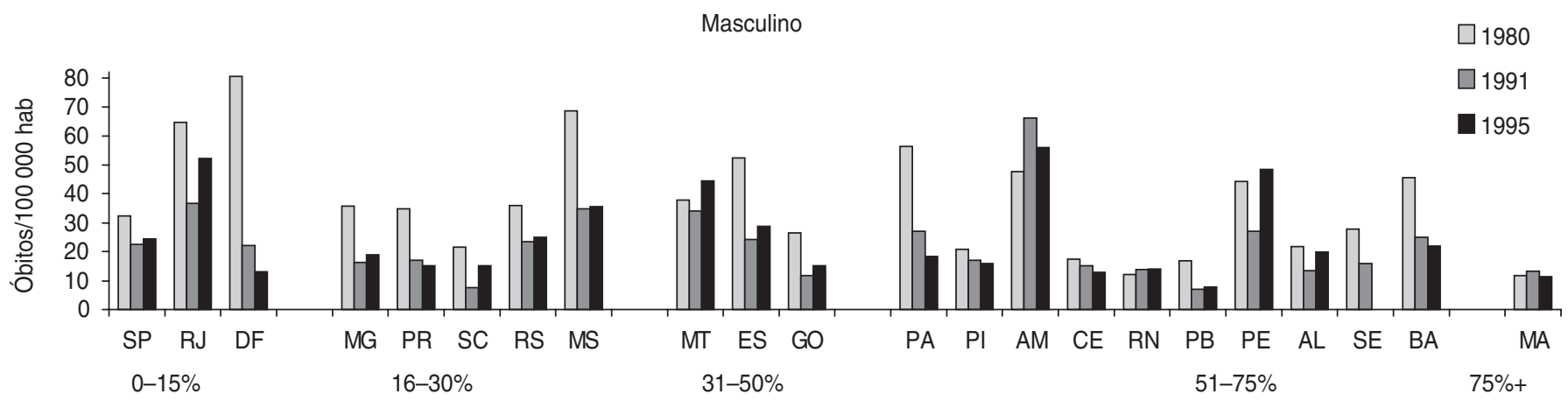

Feminino

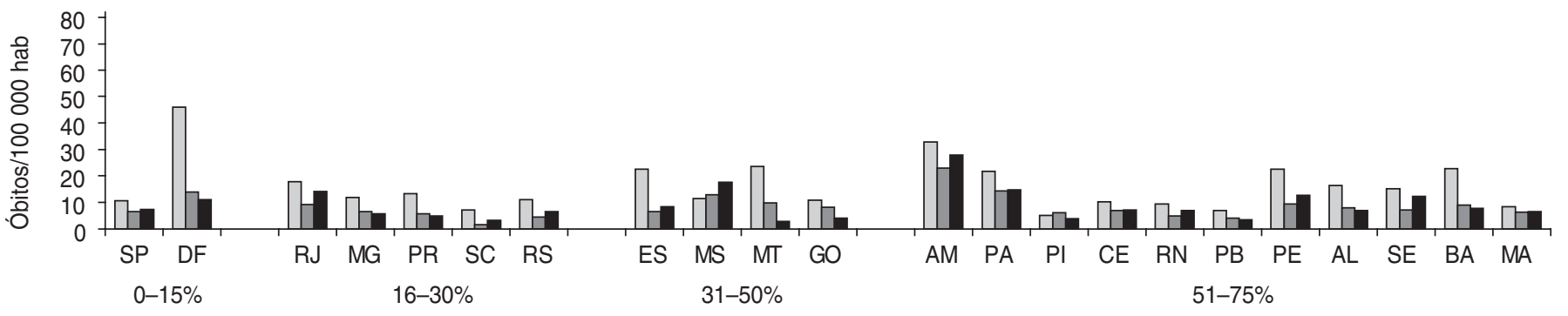

Grupos de estados conforme percentual de óbitos com causa básica desconhecida

${ }^{\text {a }} \mathrm{AL}=$ Alagoas; $\mathrm{AM}=$ Amazonas; $\mathrm{BA}=$ Bahia; $\mathrm{CE}=$ Ceará; $\mathrm{DF}=$ Distrito Federal; $\mathrm{ES}=$ Espírito Santo; $\mathrm{GO}=\mathrm{Goiás} ; \mathrm{MA}=\mathrm{Maranhão} ; \mathrm{MG}=\mathrm{Minas}$ Gerais; $\mathrm{MS}=\mathrm{Mato}$ Grosso do Sul; MT = Mato Grosso; PA = Pará; PB = Paraíba; PE = Pernambuco; PI = Piauí; PR = Paraná; RJ = Rio de Janeiro; RN = Rio Grande do Norte; RS = Rio Grande do Sul; SC = Santa Catarina; SE = Sergipe; SP = São Paulo. Não foram considerados os estados de Rondônia, Roraima, Amapá, Acre e Tocantins (Região Norte). O Estado do Tocantins não consta pois foi criado em 1988.

dicadores socioeconômicos definidos anteriormente. Os modelos finais obtidos com a aplicação do modelo de regressão linear múltipla normal propiciaram associações significativas, cujas variáveis mudam ao longo do tempo, dependendo do sexo. Os resultados encontram-se na tabela 3, onde são apresentados, para cada variável selecionada no modelo final, o coeficiente t-valor (a estatística que permite estabelecer a significância das variáveis através dos seus coeficientes), a probabilidade de significância e o coeficiente de determinação $R^{2}$ (que mede o grau de associação relativo das variáveis com a taxa de mortalidade por DIP).

\section{DISCUSSÃO}

Analisando o papel das DIPs para todas as idades no Brasil, Barreto et al. (26) afirmam que a queda na partici- pação dessas doenças no conjunto da morbi-mortalidade prevalente no país, nas duas últimas décadas, é efetivamente menor do que normalmente se tem acreditado, e que elas continuam exercendo um papel importante na mortalidade geral. A menor relevância em termos de magnitude das mortes por DIPs nos idosos no contexto das principais causas de morte não significa que o Brasil tenha controlado essas doenças, pois seus níveis ainda são elevados. Conforme o presente estudo, não há evidências de que a mortalidade por DIPs, em seu conjunto, esteja passando por um processo importante de mudança nos idosos.

Na análise da composição das doenças específicas que motivaram as mortes por DIPs, as septicemias saíram do controle agregando incertezas quanto à qualidade dos dados. $\mathrm{O}$ aumento dessas taxas na maior parte das regiões brasileiras requer investi- gações adicionais, pois se, por um lado, a importância da septicemia mostra as limitações do atendimento hospitalar para solucionar doenças complexas (27), por outro, a evolução no tempo sugere uma deterioração na verdadeira declaração da causa básica dos óbitos no Brasil. Esse fato contraria a tendência geral de melhora na qualidade dos dados em termos de cobertura e definição da causa básica, pois, na realidade, as doenças que ocasionam a septicemia é que deveriam constar como causa básica nas estatísticas de mortalidade (15). É possível que o aumento nas taxas de septicemia tenha mascarado a magnitude e o comportamento das taxas de mortalidade por outras causas de morte, e que tenha interferido nos reais níveis da tuberculose pulmonar e da tripanossomíase, gerando, para a totalidade das DIPs, uma estagnação para o período de 1980 a 1995. 
FIGURA 3. Taxas de mortalidade padronizadas da septicemia por sexo segundo classificação de causas de morte desconhecidas nas unidades da federação, Brasil, 1980 a $1995^{a}$
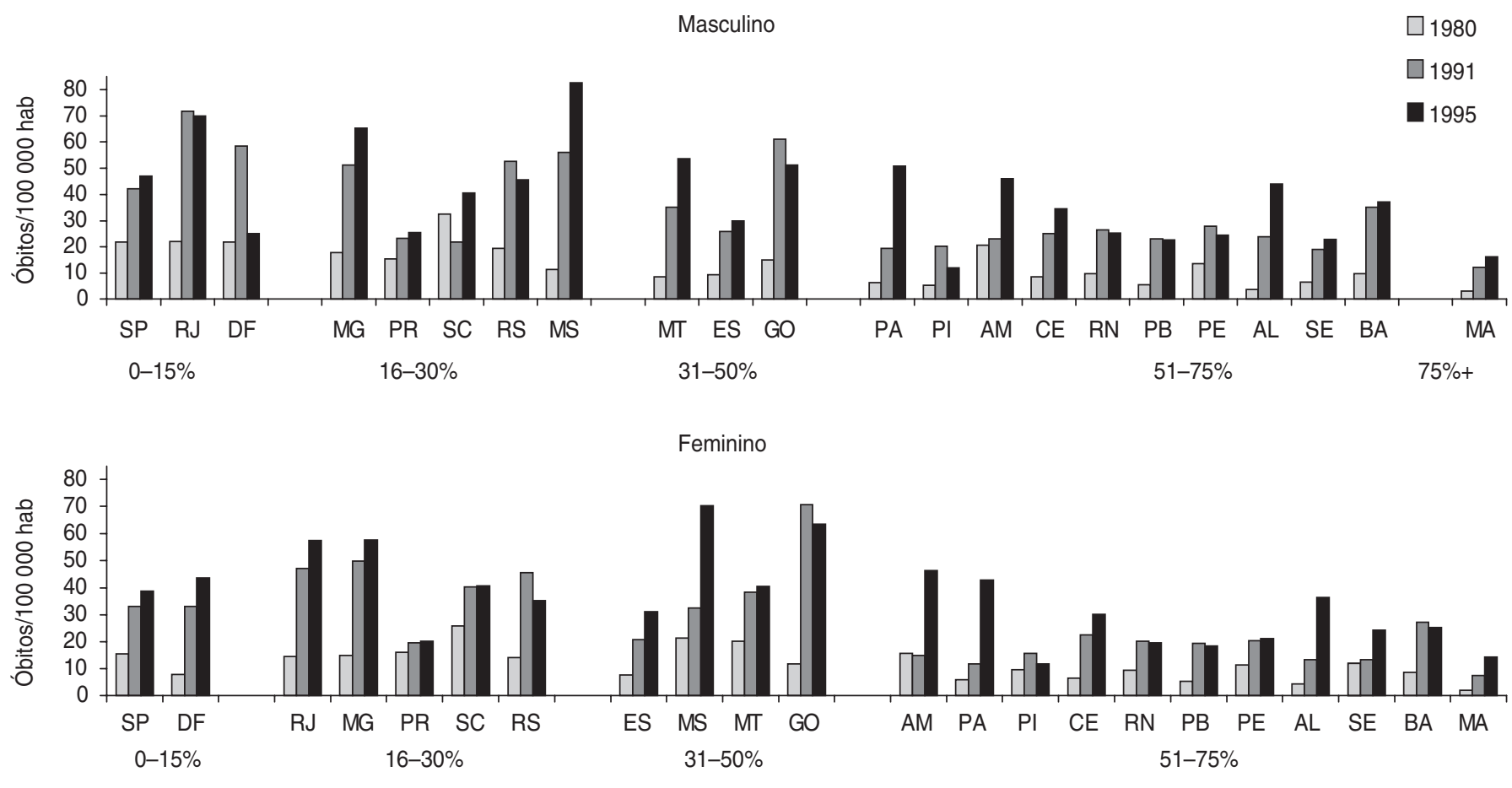

Grupos de estados conforme percentual de óbitos com causa básica desconhecida

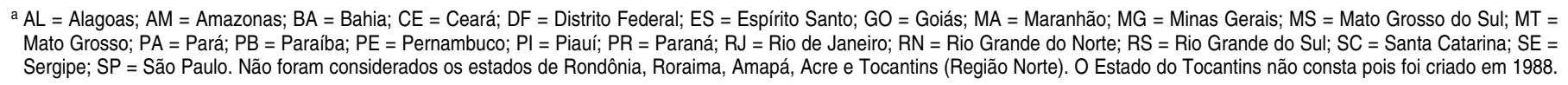

A tendência de declínio nos níveis da tuberculose pulmonar evidencia importantes ganhos em vários estados. A melhora nas condições de vida e a introdução, na década de 1940, de quimioterapia eficaz e tratamentos adequados, permitiram o controle dessa doença (28).

Seguindo a tendência da tuberculose pulmonar, existem fortes indícios de que a transmissão da doença de Chagas foi drasticamente reduzida no Brasil e controlada em algumas regiões, embora a área de transmissão da tripanossomíase ocupe um amplo espaço no território brasileiro (28). Essa redução decorreu não só das ações de programas específicos de controle, mas também do intenso processo de urbanização ocorrido no Brasil, uma vez que essa doença é mais prevalente na zona rural. Analisando este aspecto, Lima e Costa et al. (9) chamam a atenção para a importância da doença de Chagas como causa de morte entre os idosos brasileiros, a qual se deve a um efeito de coorte, conseqüência da exposição no passado à infecção pelo Trypanosoma cruzi.

Embora as desigualdades sociais entre unidades da federação predominassem nos anos 1980 e 1990, com um desnível acentuado nas mortes por DIPs $(28,29)$, os presentes resultados sugerem tendências semelhantes para vários estados do Brasil. Não há, no entanto, sinais claros de uma convergência de tendência dessas causas de morte em todo território nacional.

É interessante notar que o crescente volume de população urbana e a crescente esperança de vida ao nascer nos estados brasileiros não apresentaram associação estatística significativa com as doenças infecciosas e parasitárias. Observou-se que (embora com baixo poder explicativo para os homens), em 1980, o modelo apresentou apenas o
PIB como variável explicativa, sugerindo uma maior mortalidade em estados com maior grau de poder econômico. Em 1991, nenhuma variável explicativa apareceu na modelagem das taxas de mortalidade. Apesar de terem sido excluídos alguns estados por apresentarem problemas de desvio nos ajustes, e considerando que os problemas de sub-registro em várias regiões do Brasil eram mais graves em 1980 - o que, obviamente, levou a uma subestimação das taxas de mortalidade - é possível argumentar que a significância positiva da variável produto interno bruto per capita em 1980 tenha conduzido a uma associação também enviesada. Para 1995, o modelo final ficou composto pelas variáveis número de benefícios em manutenção pela previdência social por habitante, com coeficiente negativo, e número de leitos hospitalares por habitante, com coeficiente positivo. Pa- 
TABELA 3. Variáveis socioeconômicas associadas à mortalidade por doenças infecciosas e parasitárias na população idosa, Brasil

\begin{tabular}{|c|c|c|c|}
\hline Ano & Variável & $t$-valor & Significânciaa \\
\hline \multicolumn{4}{|l|}{ Homens } \\
\hline $1980^{b}$ & Produto interno bruto per capita & 2,17 & 5 \\
\hline $1991^{\mathrm{C}}$ & - & - & - \\
\hline $1995^{d}$ & $\begin{array}{l}\text { Número de benefícios da previdência } \\
\text { social por habitante } \\
\text { Leitos hospitalares por habitante }\end{array}$ & $\begin{array}{r}-3,38 \\
2,44\end{array}$ & $\begin{array}{l}1 \\
5\end{array}$ \\
\hline \multicolumn{4}{|l|}{ Mulheres } \\
\hline $1980^{c}$ & - & - & - \\
\hline $1991^{\mathrm{C}}$ & - & - & - \\
\hline $1995^{e}$ & $\begin{array}{l}\text { Número de benefícios da previdência } \\
\text { social por habitante }\end{array}$ & 2,31 & 5 \\
\hline
\end{tabular}

a 1 = Significativo ao nível de 1\%; 5 = significativo ao nível de $5 \%$.

${ }^{\mathrm{b}} \mathrm{DIP}=71,6+19,2$ produto interno bruto per capita; $\mathrm{R}^{2}=21,7 \%$.

c Não houve ocorrência de variáveis associadas segundo o procedimento.

${ }^{d}$ DIP $=171-19,6$ benefícios da previdência por habitante +593 leitos por habitante; $R^{2}=42,9 \%$.

e DIP = $198-9,1$ benefícios da previdência por habitante; $R^{2}=24,0 \%$.

rece incoerente que as taxas de mortalidade dos homens sejam mais elevadas naqueles estados com maior acesso a leitos hospitalares. Sem descartar a ocorrência de erros inerentes aos dados, não se pode deixar de especular que essa relação seja indiretamente explicada pela septicemia, cujos níveis de mortalidade, em 1995, foram os mais elevados entre as principais causas estudadas. Ou seja, uma maior disponibilidade de leitos poderia estar associada às chances das infecções hospitalares incidirem mais nos idosos internados.

Para as mulheres, os resultados da análise multivariada não produziram associações significativas para as variáveis de 1980 e 1991. A única variável identificada para o ano de 1995 foi número de benefícios em manutenção pela previdência social por habitante, com coeficiente negativo, sugerindo que houve uma maior taxa de mortalidade em estados com menor número de beneficiários da previdência social.
Essa associação, em consonância com aquela encontrada para os homens no mesmo ano, permite especular sobre a eficácia do aparato de prevenção e combate dos males infecciosos, que talvez não tenha conseguido acompanhar o ritmo de crescimento da demanda por cuidados da população idosa, nem atender satisfatoriamente aquelas regiões com populações menos cobertas pelos auxílios previdenciários.

Os avanços em vários campos do conhecimento, as ações e atenção à saúde, durante os 15 anos desse estudo, parecem ter surtido efeito em causas de morte isoladas, o que implicou em uma mudança no padrão da mortalidade por DIPs nos idosos no Brasil. Porém, em seu conjunto, o nível da mortalidade por DIPs não parece ter sofrido nenhuma modificação. Esses comportamentos apontam para uma ineficácia, ou estagnação, das políticas de prevenção, serviços de saúde e combate às DIPs entre os idosos. O progressivo aumento da pressão populacional por esse contingente e a manutenção dos esforços e investimentos no combate a essas doenças, em patamares similares aos exercidos durante o período de 1980 a 1995, não sugerem perspectivas de diminuição nos níveis de mortalidade por DIPs no país, pelo menos em um futuro próximo.

\section{REFERÊNCIAS}

1. Veras RP, ed. Terceira idade: um envelhecimento digno para o cidadão do futuro. Rio de Janeiro: UERJ; 1995.

2. Veras RP, Alves MI. A população idosa no Brasil: considerações acerca do uso de indicadores de saúde. Em: Minayo MC. Os muitos Brasis: saúde e população na década de 80 . São Paulo: Hucitec/Abrasco; 1995. Pp. 32037.

3. Paes NA, Silva LA. Doenças infecciosas e parasitárias no Brasil: uma década de transição. Rev Panam Salud Publica. 1999;6(2):99-109.

4. Chackiel J. La investigación sobre causas de muerte en la América Latina. Notas de Población. 1987;44:9-30.

5. Ramos LR. Growing old in São Paulo, Brazil: assessment of health status and social support of elderly people from different socioeconomic strata living in the community [tese]. London: London School of Hygiene blica. 1993;27(2):87-94. and Tropical Medicine, University of London; 1987.

6. Veras RP. A survey of the health of elderly people in Rio de Janeiro, Brazil [tese]. London: Division of Psychiatry, United Medical and Dental School, Guy's Hospital, University of London; 1992.

7. Ramos LR, Rosa TE, Oliveira ZM, Medina $\mathrm{MC}$, Santos FR. Perfil do idoso em área metropolitana na região sudeste do Brasil: resultado de inquérito domiciliar. Rev Saude Pu-

8. Coelho Filho JM, Ramos LR. Epidemiologia do envelhecimento no nordeste do Brasil: resultados de inquérito domiciliar. Rev Saude Publica. 1999;33(5):445-53.

9. Lima e Costa MFFL, Uchoa E, Guerra HL, Firmo JOA, Vidigal PG, Barreto SM. The Bambui Health and Ageing Study (BHAS). Methodological approach and preliminary re- sults of a population based cohort study of the elderly in Brazil. Rev Saude Publica. 2000; 34(2):126-35.

10. Brasil, Ministério da Saúde. Sistema de informações sobre mortalidade, 1979-1993. Brasília: CENEPI; 1996.

11. Fundação Instituto Brasileiro de Geografia e Estatística. IX Recenseamento geral do Brasil, 1980, censo demográfico (Brasil e estados). Rio de Janeiro: IBGE; 1983.

12. Fundação Instituto Brasileiro de Geografia e Estatística. X Recenseamento geral do Brasil, 1991, censo demográfico (Brasil e estados). Rio de Janeiro: IBGE; 1995.

13. Fundação Instituto Brasileiro de Geografia e Estatística. Contagem da população - 1996. Disponível em: http://www.ibge.gov.br Acessado em março de 2004.

14. Paes NA, Albuquerque MEE. Avaliação da qualidade dos dados populacionais e cober- 
tura dos registros de óbitos para as regiões brasileiras. Rev Saude Publica. 1999;33(1):3343.

15. Mello Jorge MH. Como morrem nossos jovens. Em: Comissão Nacional de População e Desenvolvimento. Jovens acontecendo na trilha das políticas públicas. Brasília: CNPD; 1998. Pp. 209-92.

16. Cerqueira CA. Mortalidade por causas nas capitais brasileiras e suas associações com indicadores socioeconômicos [dissertação]. Recife: Departamento de Estatística, Universidade Federal de Pernambuco; 1998.

17. Sichieri R, Lolio CA, Correia VR, Everhart JE. Geographical patterns of proportionate mortality for the most common causes of death in Brazil. Rev Saude Publica. 1992;26(6):424-30.

18. Preston SH. Mortality patterns in national populations: with special reference to recorded causes of death. London: Academic Press; 1976.

19. Paes NA. Mortalidade adulta no Brasil: tendências, diferenciais e causas. Brasília: CNPq; 1999
20. Programa das Nações Unidas para o Desenvolvimento, Instituto de Pesquisa Econômica Aplicada, Fundação João Pinheiro, Instituto Brasileiro de Geografia e Estatística. Desenvolvimento humano e condições de vida: indicadores brasileiros. Brasília: PNUD; 1998.

21. Fundação Instituto Brasileiro de Geografia e Estatística. Anuário estatístico do Brasil: 1993. Rio de Janeiro: IBGE; 1994

22. Fundação Instituto Brasileiro de Geografia e Estatística. Anuário estatístico do Brasil: 1996. Rio de Janeiro: IBGE; 1997.

23. Fundação Instituto Brasileiro de Geografia e Estatística. Estatísticas de saúde: assistência médico-sanitária. Rio de Janeiro: IBGE; 1992.

24. Searle SR. Linear models. Nova York: J. Wiley \& Sons; 1971.

25. Draper NR, Smith H. Applied regression analysis. New York: J. Wiley \& Sons; 1981.

26. Barreto ML, Carmo EH, Santos CAS, Ferreira LDA. Emergentes, re-emergentes e permanecentes: tendências recentes das doenças infecciosas e parasitárias no Brasil. Inf Epidemiol SUS. 1996;3:7-17.
27. Sabrosa PC, Kawa H, Campos WSQ. Doenças transmissíveis: ainda um desafio. Em: Minayo MC, org. Os muitos Brasis, saúde e população na década de 80. São Paulo: Hucitec/Abrasco; 1995.

28. Waldman EA, Silva LJ, Monteiro CA. Trajetória das doenças infecciosas: da eliminação da poliomielite à reintrodução da cólera. Em: Monteiro CA. Velhos e novos males da saúde no Brasil: a evolução do país e de suas doenças. São Paulo: Hucitec NUPENS/Universidade de São Paulo; 1995. Pp. 17-30.

29. Chaimowick F. A saúde dos idosos brasileiros às vésperas do século XXI: problemas, projeções e alternativas. Rev Saude Publica. 1997; 31(2):184-200.

Manuscrito recebido em 17 de fevereiro de 2003. Aceito em versão revisada em 8 de janeiro de 2004.

ABSTRACT Objective. To describe the mortality due to infectious and parasitic diseases in persons over 65 years of age in all of Brazil and in individual states and to study the association between that mortality due to these causes and specific socioeconomic variables over the period from 1980 to 1995.

\section{Mortality due to infectious and parasitic diseases in the elderly in Brazil}

Methods. Data were obtained from Brazil's national Mortality Information System. Standardized mortality rates were calculated for tuberculosis, Chagas' disease, and sepsis. The relative contribution of these causes of death to overall mortality was also calculated. The study also analyzed the relationship between mortality rates and gender and the following socioeconomic variables: per capita gross domestic product; number of hospital beds per inhabitant; proportion of the total population living in urban areas; number of benefits provided per capita by the social welfare system; number of deaths reported for every 100 hospitalizations; and life expectancy at birth. A normal multiple linear regression model was used for this analysis.

Results. There were no significant changes in the proportion of deaths due to infectious and parasitic diseases in the elderly population during the period studied, regardless of sex. However, for both men and women there was a sizeable decrease in the impact of tuberculosis and Chagas' disease, while that of sepsis appeared to increase. According to the results of the ecological analysis, socioeconomic indicators had little power to explain the differences in mortality patterns seen in the different states of the country.

Conclusions. Our results suggest that policies to prevent and control infectious and parasitic diseases among the elderly in Brazil have stagnated. Given this situation and the progressive increase in the elderly population, mortality due to infectious and parasitic diseases is not likely to decrease in this age group, at least in the near future. 